Europhys. Lett., 58 (6), pp. 919-925 (2002)

\title{
Structure and tracer-diffusion in quasi-two-dimensional and strongly asymmetric magnetic colloidal mixtures
}

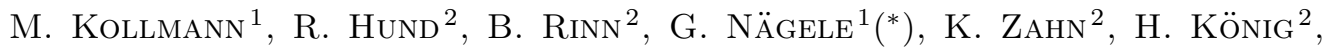

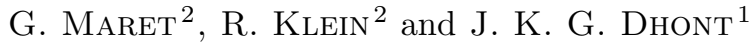 \\ 1 Institut für Festkörperforschung, Teilinstitut Weiche Materie \\ Forschungszentrum Jülich - D-52425 Jülich, Germany \\ 2 Fachbereich Physik, Universität Konstanz \\ Postfach 5560, D-78457 Konstanz, Germany
}

(received 7 January 2002; accepted in final form 20 March 2002)

PACS. 82.70.Dd - Colloids.

PACS. 05.40. Jc - Brownian motion.

PACS. 05.10.Gg - Stochastic analysis methods (Fokker-Planck, Langevin, etc.).

\begin{abstract}
We study theoretically and experimentally static and diffusional properties of a strongly asymmetric binary mixture of super-paramagnetic colloids confined to an air-water interface. The colloids interact via repulsive dipolar forces, induced by a magnetic field applied perpendicular to the planar interface. Brownian dynamics (BD) computer simulations are employed to analyse the microstructure and the tracer-diffusion of both components. The profound interaction asymmetry leads to unusual features in the partial pair distribution functions, and to significantly enhanced tracer-diffusion. We find good agreement between our experimental data and the computer simulation results.
\end{abstract}

Introduction. - A lot of effort has been devoted over the past years to study the structural and dynamical properties of quasi-two-dimensional (Q2D) model dispersions of monodisperse super-paramagnetic colloidal spheres confined to a liquid-gas interface and interacting by induced repulsive dipolar forces [1-4]. These model systems are of particular interest both from the experimental and theoretical point of view, since the interaction potential is well characterised and the two-dimensional trajectories can be followed over an extended time range using video imaging. The interaction strength can be precisely tuned by the applied magnetic field. This allows to study in great detail interesting phenomena like the freezing and melting transition via an intermediate hexatic phase of quasi-long-range orientational order [5].

While static properties are independent of the solvent flow, it is known that the dynamics of Q2D monodisperse systems is strongly affected by the solvent-mediated hydrodynamic interactions (HI). This gives rise to remarkable effects like the divergence of certain shorttime diffusional transport coefficients $[3,4,6]$, and the enhancement or de-enhancement of

$\left(^{*}\right)$ E-mail: g.naegele@fz-juelich.de

(C) EDP Sciences 
self-diffusion. For short-range particle forces it has been found that HI causes a slowing-down of self- and collective diffusion in two- and three-dimensional dispersions [7]. For long-range repulsive interactions, there is instead a hydrodynamic enhancement of self-diffusion, as shown recently for magnetic Q2D systems [1-4], wall-confined charged-stabilised Q2D colloids [6], and also for three-dimensional charge-stabilised dispersions [8,9]. Q2D systems with long-range repulsive interactions show further an interesting dynamic scaling behaviour. As a consequence of dynamic scaling, the freezing point can be characterised by various equivalent dynamic freezing criteria in terms of long-time self- and collective-diffusion properties $[3,4,10,11]$.

While quite some work has been done on monodisperse Q2D systems, very little is known on binary Q2D dispersions. Two-component Q2D and three-dimensional systems exhibit additional interesting features not present in monodisperse systems, like two-stage vitrification for strongly asymmetric components, where the more strongly interacting component becomes structurally arrested first, and interdiffusion processes. Furthermore, two-component systems do not show simple scaling behaviour, thus invalidating the static and dynamic freezing criteria and the generalised Stokes-Einstein relations obeyed approximately in the monodisperse case.

In this letter, we study the microstructure and the tracer-diffusion of fluid binary Q2D dispersions of super-paramagnetic particles at a water-air interface, where the two components are very different in the strengths of the dipolar magnetic repulsion. Particular focus is given to reveal the influence of $\mathrm{HI}$ on the tracer-diffusion of the particles. For this purpose we compare $\mathrm{BD}$ results for the reduced mean-squared displacements of both components with and without far-field HI included with corresponding experimental results on micrometer-sized particles obtained from video imaging. As we will show subsequently, the good agreement between our simulations with $\mathrm{HI}$ and the experiments clearly demonstrates the strong hydrodynamic coupling of the particles. The BD scheme with HI can thus be faithfully used even for a quantitative exploration of strongly asymmetric two-dimensional colloidal fluids.

Experimental setup. - The characterisation, preparation and measurements of twocomponent dispersions of magnetic colloids follow essentially the procedure described in [5]. Therefore we only briefly summarise the essentials: experiments have been performed on binary mixtures of spherical super-paramagnetic colloids of particle radii $a_{1}=2.35 \mu \mathrm{m}$, $a_{1}=1.4 \mu \mathrm{m}$ and mass densities $\rho_{1}=1.6 \mathrm{~kg} / \mathrm{dm}^{3}, \rho_{2}=1.3 \mathrm{~kg} / \mathrm{dm}^{3}$ confined by gravity to a water-air interface of a hanging drop in a top-sealed ring. The flatness of the water-air interface with diameter of $8 \mathrm{~mm}$ is controlled within a maximal height variation of $\pm 1 \mu \mathrm{m}$. The particle monolayer at the interface consists of about $10^{5}$ particles, out of which about $10^{3}$ particles near the centre of the interface are tracked in time steps of $\Delta t=0.2 \mathrm{~s}$ using realspace video imaging. Since an isolated particle needs about $100 \mathrm{~s}$ to diffuse a distance equal to its own diameter, more than a one-day measurement is required to monitor each of the mean-squared displacements discussed in this work. A weak magnetic field $B$ applied perpendicular to the interface induces magnetic dipole moments, $M_{\alpha}=\chi_{\alpha} B$, in the $\mathrm{Fe}_{2} \mathrm{O}_{3}$-doped super-paramagnetic particles of component $\alpha \in\{1,2\}$, with effective magnetic susceptibilities $\chi_{1}=6.2 \cdot 10^{-11} \mathrm{Am}^{2} / \mathrm{T}$ and $\chi_{2}=6 \cdot 10^{-12} \mathrm{Am}^{2} / \mathrm{T}$. The long-range magnetic repulsion between two spheres of components $\alpha$ and $\beta$ a distance $r$ apart in the interface is thus well described by the dipolar pair potential $u_{\alpha \beta}(r)$, with

$$
\frac{u_{\alpha \beta}(r)}{k_{\mathrm{B}} T}=\frac{\Gamma_{\alpha} \Gamma_{\beta}}{x^{3}}
$$

Here, $x=r / r_{0}$ is a reduced distance, with $r_{0}=n^{-1 / 2}$ denoting the geometric mean particle distance related to the total area number density, $n=n_{1}+n_{2}$, of both components. In eq. (1), we have further introduced the dimensionless coupling amplitude $\Gamma_{\alpha}=\left[\mu_{0} /\left(4 \pi k_{\mathrm{B}} T r_{0}^{3}\right)\right]^{1 / 2} M_{\alpha}$ 
of component $\alpha$ with $\Gamma_{\alpha} \Gamma_{\beta}$ quantifying the interaction energy between two particles of components $\alpha$ and $\beta$, respectively, a distance $r_{0}$ apart from each other. We point out that the interaction energy between two 1-particles separated a distance $r_{0}$ is approximately $10^{2}$ times larger than the interaction energy of two 2-particles at the same distance.

Brownian dynamics simulations. - The BD finite-difference equation describing the inplane displacement of $N$ spherical Brownian particles immersed in a fluid of viscosity $\eta$ during a time step $\Delta t$ is given by [12]

$$
\boldsymbol{R}_{i}(t+\Delta t)-\boldsymbol{R}_{i}(t)=\sum_{j=1}^{N}\left[\beta \boldsymbol{D}_{i j}\left(\boldsymbol{R}^{N}\right) \cdot \boldsymbol{F}_{j}^{P}+\nabla_{j} \cdot \boldsymbol{D}_{i j}\left(\boldsymbol{R}^{N}\right)\right] \Delta t+\Delta \boldsymbol{X}_{i}+\mathcal{O}\left(\Delta t^{2}\right) .
$$

Here, $\boldsymbol{R}^{N}=\left\{\boldsymbol{R}_{1}, \ldots, \boldsymbol{R}_{N}\right\}$, with $\boldsymbol{R}_{i}(t)$ denoting the position vector pointing to the centre of sphere $i$ at time $t, \boldsymbol{F}_{j}^{P}=-\nabla_{j} \sum_{k<i}^{N} u_{i k}\left(\boldsymbol{R}_{i}-\boldsymbol{R}_{k}\right)$ is the total direct force on particle $j$ due to all other $N-1$ particles, and $\Delta \boldsymbol{X}_{i}$ is a random displacement vector of particle $i$ originating from the collisions with solvent particles. The random displacement $\Delta \boldsymbol{X}_{i}$ is sampled from a Gaussian distribution with zero mean, $\left\langle\Delta \boldsymbol{X}_{i}\right\rangle=0$, and covariance matrix $\left\langle\Delta \boldsymbol{X}_{i} \Delta \boldsymbol{X}_{j}\right\rangle=2 \boldsymbol{D}_{i j}\left(\boldsymbol{R}^{N}\right) \Delta t$.

The brackets indicate a canonical (NVT) equilibrium ensemble average and we employ here the polyadic tensor notation. The solvent-mediated HI between particles $i$ and $j$ are accounted for in the BD algorithm through the hydrodynamic diffusivity tensors $\boldsymbol{D}_{i j}\left(\boldsymbol{R}^{N}\right)$ which depend, in principle, on the configuration $\boldsymbol{R}^{N}=\left\{\boldsymbol{R}_{1}, \ldots, \boldsymbol{R}_{N}\right\}$ of all $N$ sphere centres at time $t$. For the strongly repelling and rather dilute dispersed colloidal particles under consideration, the HI are dominated by their pairwise additive long-range part. Therefore, we can treat $\boldsymbol{D}_{i j}$ within good accuracy on the Rotne-Prager (RP) approximation level, i.e. [7,13]

$$
\boldsymbol{D}_{i j}\left(\boldsymbol{R}^{N}\right) \approx D_{i}^{0, \mathrm{f}}\left[\delta_{i j} \mathbf{1}+\left(1-\delta_{i j}\right) \boldsymbol{T}_{i j}^{\mathrm{RP}}\left(\boldsymbol{R}_{i}-\boldsymbol{R}_{j}\right)\right],
$$

where $\mathbf{1}$ is the unit tensor, $\delta_{i j}$ is the Kronecker symbol, and $D_{i}^{0, \mathrm{f}}$ denotes the in-plane freediffusion coefficient of a sphere $i \in\{1, \ldots, N\}$. The Rotne-Prager tensor, $\boldsymbol{T}_{i j}^{\mathrm{RP}}$, reads explicitly $[7,13]$

$$
\boldsymbol{T}_{i j}^{\mathrm{RP}}(\boldsymbol{r})=a_{i}\left[\frac{3}{4 r}[\mathbf{1}+\hat{\boldsymbol{r}} \hat{\boldsymbol{r}}]+\frac{1}{4} \frac{a_{i}^{2}+a_{j}^{2}}{r^{3}}[\mathbf{1}-3 \hat{\boldsymbol{r}} \hat{\boldsymbol{r}}]\right],
$$

with $\hat{\boldsymbol{r}}=\boldsymbol{r} / r$. The in-plane free-diffusion coefficient $D_{i}^{0, \mathrm{f}}$ is somewhat larger than the Stokesean translational diffusion coefficient, $D_{i}^{0}=k_{\mathrm{B}} T /\left(6 \pi \eta a_{i}\right)$, of an isolated sphere in an unbounded fluid. Since we consider only tracer diffusion properties normalised by the corresponding $D_{i}^{0, \mathrm{f}}$, the actual values of the in-plane free-diffusion coefficients are here of no concern.

The finite-difference equation (2) is solved using an Euler scheme with typically 200 particles. For calculating the dipolar forces $\boldsymbol{F}_{j}^{P}$, the simulation square is periodically repeated in two dimensions. The long-range nature of HI inherent in $\boldsymbol{D}_{i j}$ is accounted for by employing a two-component Ewald-type summation technique on the Rotne-Prager level developed by Beenakker [14]. To integrate eq. (2) we have used a fixed time step $2 \times 10^{-4} \tau_{1}$, where $\tau_{1}=1 /\left(n D_{1}^{0, \mathrm{f}}\right)$ is the time needed for a free particle of component 1 to diffuse approximately an interparticle distance $n^{-1 / 2}$. After equilibration several thousand time steps were generated by the BD algorithm to obtain particle mean-squared displacements and radial distribution functions.

The BD simulation code with HI included has been extensively tested for monodisperse super-paramagnetic Q2D systems in comparison with experimental data [1], and alternative BD simulation codes [6]. Very good agreement has been found in all cases considered. 

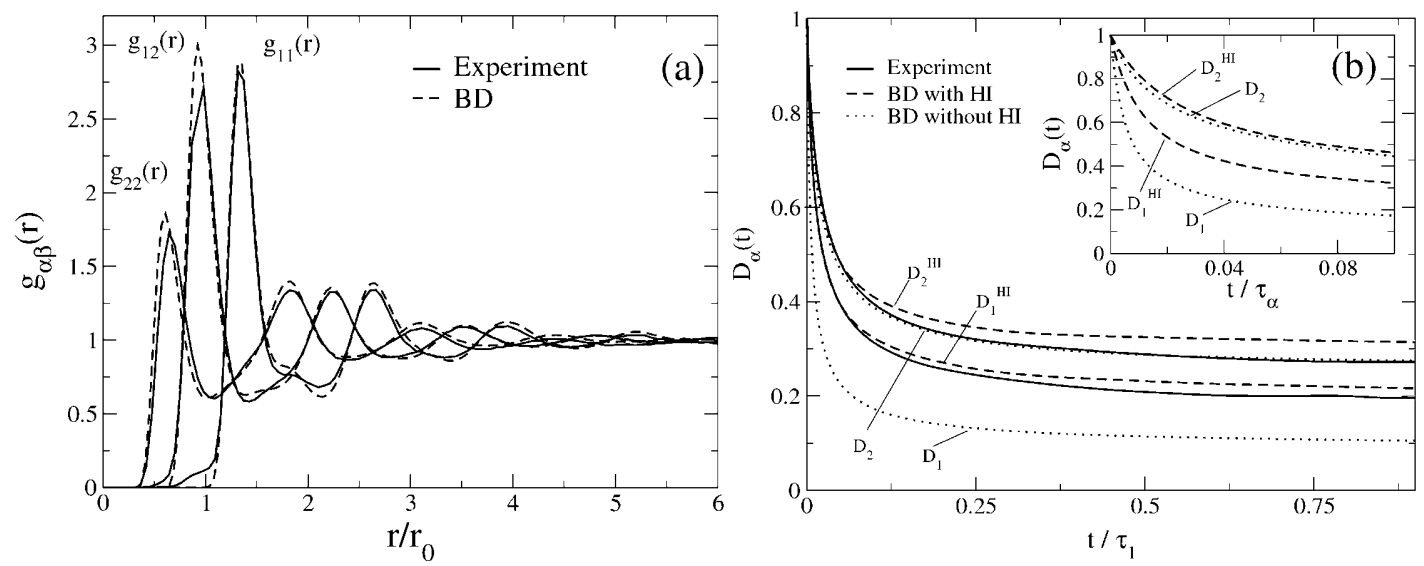

Fig. 1 - Binary suspension of super-paramagnetic particles with system parameters $B=1.26 \mathrm{mT}$, $n=n_{1}+n_{2}=4.08 \cdot 10^{-3} \mu \mathrm{m}^{-2}$ and $n_{1} / n_{2}=1.043$. The values for the magnetic susceptibilities, $\chi_{\alpha}$, and particle radii, $r_{\alpha}$, are given in the text. (a) Partial radial distribution functions, $g_{\alpha \beta}(r)$, vs. reduced distance $r / r_{0}$. (b) BD simulation results with and without $\mathrm{HI}$ and experimental results for the reduced tracer-diffusion functions, $D_{\alpha}(t)$, vs. reduced time $t / \tau_{1}$, with $\tau_{1}=r_{0}^{2} / D_{1}^{0, \mathrm{f}}$. Inset: $\mathrm{BD}$ results for $D_{\alpha}(t)$ vs. $t / \tau_{\alpha}$.

Discussion. - We first assess the reliability of the BD scheme by comparing the numerically determined partial pair correlation functions, $g_{\alpha \beta}(r)$, with the video imaging results. Here, $n_{\beta} g_{\alpha \beta}(r)$ is the average number density of $\beta$-type particles a distance $r$ apart from the centre of a given $\alpha$-particle. Two different binary mixtures of magnetic particles are considered. The two mixtures share the same values for the magnetic susceptibilities $\chi_{\alpha}$ and radii $a_{\alpha}$. The first system is characterised by a total number density of $n=n_{1}+n_{2}=4.08 \cdot 10^{-3} \mu \mathrm{m}^{-2}$, with number ratio $n_{1} / n_{2}=1.043$. The externally applied magnetic field strength is $B=1.26 \mathrm{mT}$, with coupling amplitudes $\Gamma_{1}=38.9$ and $\Gamma_{2}=0.493$ for water at room temperature. Note that the excluded-volume interactions are of no relevance due to the strong magnetic repulsion, even of the type 2 particles. Simulation and experimental results for the three partial radial distribution functions, $g_{\alpha \beta}(r)$, of the first mixture are shown in fig. 1a.

The partial radial distribution functions of the second mixture are depicted in fig. 2a. Here, the system parameters are $n=n_{1}+n_{2}=2.31 \cdot 10^{-3} \mu \mathrm{m}^{-2}, n_{1} / n_{2}=0.313$ and $B=2.95 \mathrm{mT}$. This results in a mean particle distance $r_{0}=20.8 \mu \mathrm{m}$, and in partial coupling amplitudes $\Gamma_{1}=91.8$ and $\Gamma_{2}=1.16$. While this system is more dilute than the first one, the particles are more strongly coupled due to the higher magnetic field strength. As seen, the $g_{\alpha \beta}(r)$ of both systems exhibit pronounced oscillations with well-developed next-neighbor shells, at distances comparable to $r_{0}$. We further observe the expected ordering $r_{11}>r_{12}>r_{22}$, where $r_{\alpha \beta}$ is the location of the principal maximum of $g_{\alpha \beta}(r)$. In contrast, the BD results of figs. 1a and 2a do not show the usual ordering $g_{11}>g_{12}>g_{22}$ of the principal peak heights of the $g_{\alpha \beta}(r)$. Such a monotonic increase of the peak heights with increasing coupling strength has been observed, e.g., in three-dimensional binary dispersions of neutral and charged colloidal spheres [7].

Unlike fig. 1a, there is a peculiar feature in the radial distribution functions of the second system, namely the splitting of the primary peak of $g_{11}(r)$ into two peaks. This peak-splitting of the 1-1 pair distribution function arises from the stronger magnetic coupling of both components, in combination with the large interaction asymmetry $\left(\Gamma_{1} / \Gamma_{1}\right)^{2} \approx 10^{2}$ and the specific values of the partial number densities. The twin peaks of nearly equal height appear in the 

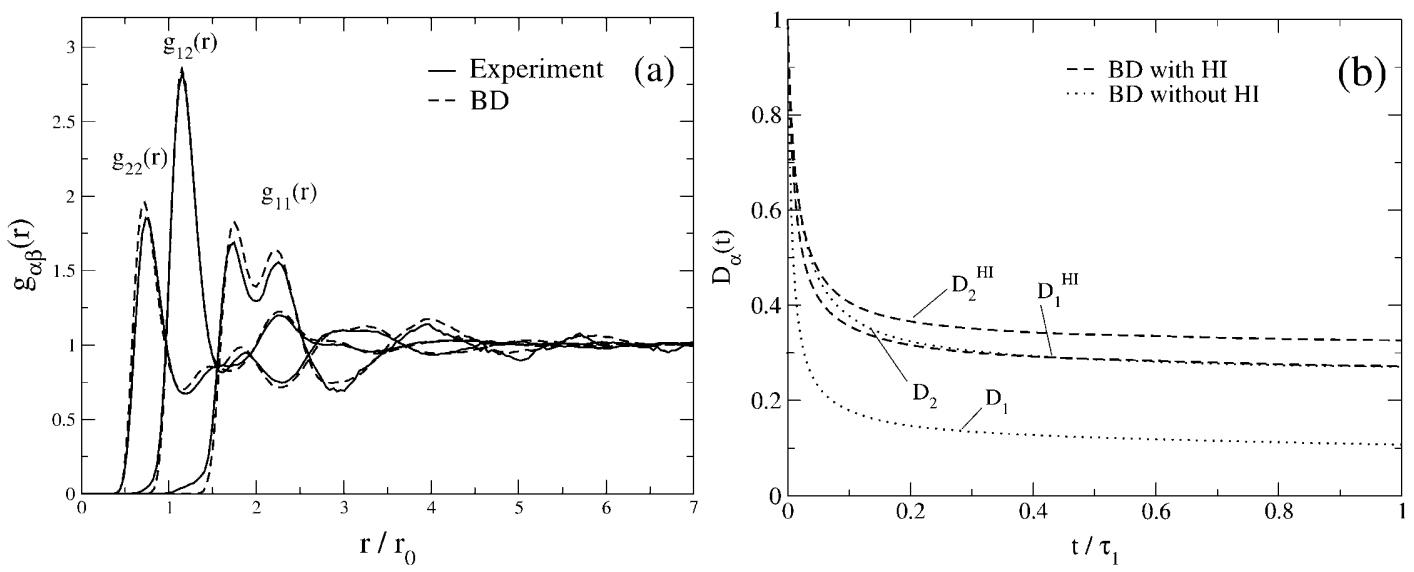

Fig. 2 - Binary suspension with system parameters $B=2.95 \mathrm{mT}, n=n_{1}+n_{2}=2.31 \cdot 10^{-3} \mu \mathrm{m}^{-2}$, $n_{1} / n_{2}=0.313$ and same magnetic susceptibilities and particle radii as in fig. 1. (a) Partial radial distribution functions, $g_{\alpha \beta}(r)$, vs. reduced distance $r / r_{0}$. (b) BD simulation results for the reduced tracer-diffusion function, $D_{\alpha}(t)$, vs. reduced time $t / \tau_{1}$.

$g_{11}(r)$ of fig. 2a since there are two concurring sets of configurations of nearly equal statistical weight: the innermost next-neighbor shell formed around a 1-particle, which includes 1-particles as characterised by the first peak in $g_{11}(r)$, contains a larger fraction of 2-particles than the more distant second subshell, characterised by the second peak in $g_{11}(r)$. This is consistent with the fact that the second primary minimum of $g_{12}(r)$, and the second primary maximum of $g_{22}(r)$ are located close to the second maximum of $g_{11}(r)$. There is nearly quantitative agreement between the computer simulation and the experimental $g_{\alpha \beta}(r)$, despite the strong interaction asymmetries. The remaining differences in the $g_{\alpha \beta}(r)$ can be attributed to remaining statistical errors in the video imaging and simulation data evaluation. Moreover, there might be significant polydispersity in the magnetic moments of the 2-particles whose distribution is unknown and thus not included in the BD simulations.

The dipolar magnetic pair potential in eq. (1) contains no intrinsic length scale. Provided the magnetic field is so strong that the particles do not experience their physical hard cores, $r_{0}=n^{-1 / 2}$ is the only physically relevant static length scale. It follows that magnetic Q2D mixtures with identical sets of coupling amplitudes $\left\{\Gamma_{\alpha}\right\}$ are statistically equivalent, i.e. they have identical $g_{\alpha \beta}(r)$ as functions of reduced distance $r / r_{0}$. An analogous conclusion can be drawn for the dynamics as described by the overdamped Langevin equation, eq. (2): without HI, i.e. when $\boldsymbol{D}_{i j}=D_{i}^{0, \mathrm{f}} \delta_{i j} \mathbf{1}$, systems of equal coupling amplitudes and equal sets of particle size ratios $\left\{a_{\alpha} / a_{1}\right\}$ are dynamically equivalent, i.e. they have identical reduced tracer diffusion functions

$$
D_{\alpha}(t)=\frac{W_{\alpha}(t)}{t D_{\alpha}^{0, \mathrm{f}}}
$$

as functions of reduced time $t / \tau_{\alpha}$, with $\tau_{\alpha}=r_{0}^{2} / D_{\alpha}^{0, \mathrm{f}}$. With far-field HI included according to eqs. (3) and (4), there is the more stringent condition of equal sets of size ratios $\left\{a_{\alpha} / r_{0}\right\}$ to enforce statistical equivalence. Here $W_{\alpha}(t)=\left\langle\left[\boldsymbol{R}_{\alpha}(t)-\boldsymbol{R}_{\alpha}(0)\right]^{2}\right\rangle / 4$ is the two-dimensional mean squared displacement of $\alpha$-type particles.

The systems in figs. 1 and 2 are obviously far from being stochastically equivalent due to the different values for $\Gamma_{\alpha}$, although the size ratio $a_{1} / a_{2}$ is the same for both cases. The 


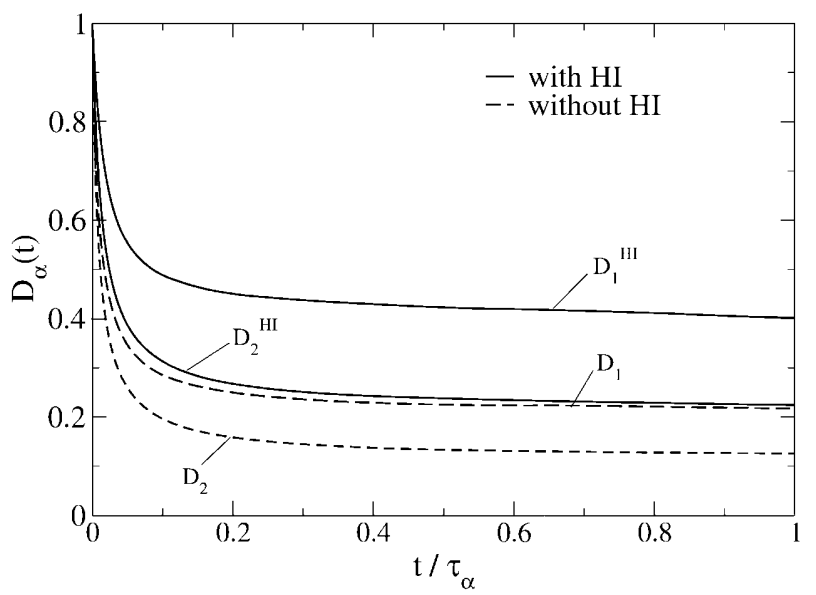

Fig. 3 - BD results of $D_{\alpha}(t)$ vs. $\tau_{\alpha}$ for a symmetric binary mixture with system parameters $\Gamma_{1}=$ $\Gamma_{2}=8, a_{1}=0.15 r_{0}, a_{2}=a_{1} / 2$, and $n_{1}=n_{2}$.

occurrence of several characteristic lengths, i.e. $\left\{a_{\alpha}\right\}$ and $r_{0}$, in colloidal mixtures renders their dynamics more complicated than in the one-component case. For mixtures, there is no simple static and dynamic scaling behaviour as observed in one-component dispersions of strongly repelling particles $[3,4,10,11]$. As a consequence the simple freezing criteria known for one-component systems do not apply to colloidal mixtures.

BD simulation results with and without HI for the reduced tracer-diffusion functions, $D_{\alpha}(t)$, of both systems are shown in figs. $1 \mathrm{~b}$ and $2 \mathrm{~b}$. Figure $1 \mathrm{~b}$ also includes experimental results for the $D_{\alpha}(t)$. In the definition of $D_{\alpha}(t)$ (cf. eq. (5)) a trivial particle size dependence of the partial mean-squared displacements $W_{\alpha}$ through the $D_{\alpha}^{0, \mathrm{f}}$ has been divided out. The significant hydrodynamic enhancement of tracer-diffusion is clearly observed for both components. It is interesting to see that the hydrodynamic enhancement of the tracer-diffusion function, $D_{1}(t)$, of the more strongly interacting particles is much stronger than for the function, $D_{2}(t)$, of the more weakly interacting component. The strong dependence of the diffusion enhancement on the strength of the magnetic moments of the particles is not shared by monodisperse systems. For the latter, the hydrodynamic increase in $D(t)$ is only weakly dependent on the coupling parameter $\Gamma$ (cf. ref. [2]). The experimental values of $D_{\alpha}(t)$ are, for larger correlations times $t$, somewhat smaller than the BD results without HI. This observation holds true also for the monodisperse case. It is probably due to our approximate far-field treatment of the diffusivity matrix $\boldsymbol{D}_{i j}\left(\boldsymbol{R}^{N}\right)$. With increasing concentration and decreasing coupling, higher-order terms in the multipole expansion of $\boldsymbol{D}_{i j}\left(\boldsymbol{R}^{N}\right)$ come into play which have the tendency to reduce the diffusion enhancement originating from the Rotne-Prager contribution to the diffusivity tensors. As seen, the BD results with $\mathrm{HI}$ for the difference $\left|D_{1}(t)-D_{2}(t)\right|$ of strongly and weakly magnetic particles are quite close to the experimental ones. In contrast, the difference between the $D_{1}(t)$ and $D_{2}(t)$ without $\mathrm{HI}$ is typically twice as large as with HI (cf. figs. $1 \mathrm{~b}$ and $2 \mathrm{~b}$ ). As can be expected, the tracer-diffusion function $D_{1}(t)$ of the stronger magnetic particles decays initially much faster than $D_{2}(t)$. The fast decay of $D_{1}(t)$ compared to $D_{2}(t)$ becomes more visible when each of the two $D_{\alpha}(t)$ is plotted vs. its own reduced time $t / \tau_{\alpha}$ (cf. the inset of fig. 1b), thus correcting for the larger size and Stokesian friction of component 1-particles. There are unfortunately no experimental data available to compare the computer simulation results in fig. 2b. However, similar good agreement between BD 
simulation and experiment can be expected as for the system discussed in fig. 1 .

Having discussed binary systems of strong interaction asymmetry, we explore finally the opposite case of interacting monodisperse mixtures. In fig. 3, we show BD results with/without HI of $D_{\alpha}(t)$ vs. $\tau_{\alpha}$ for a binary mixture, where both components are of same number density and magnetic coupling strength. The only difference is that the 1-particles are twice as large as the 2-particles (i.e., $D_{1}^{0, \mathrm{f}}=0.5 D_{2}^{0, \mathrm{f}}$ ). Whereas $g_{11}(r)=g_{12}(r)=g_{22}(r)$, the $D_{1}(t)$ of the larger and less mobile 1-particles decays more slowly towards a substantally larger longtime limit $D_{1}(\infty)>D_{2}(\infty)$. The present system shows a remarkably strong hydrodynamic enhancement of tracer-diffusion. To conclude, we have investigated the influence of strong interaction asymmetry, particle size effects and hydrodynamic interaction effects on the statics and dynmamics of binary Q2D dispersions. We have shown that the BD results are in good accord with experimental data.

$$
* * *
$$

This research work was funded by the Deutsche Forschungsgemeinschaft (SFB 513).

\section{REFERENCES}

[1] Zahn K., Mendez-Alcaraz J. M. and Maret G., Phys. Rev. Lett., 79 (1997) 175.

[2] Rinn B., Zahn K., Maass P. and Maret G., Europhys. Lett., 46 (1999) 537.

[3] Pesché R., Kollmann M. and Näegele G., J. Chem. Phys., 114 (2001) 114.

[4] Nägele G., Kollmann M., Pesché R. and Banchio A., to be published in Mol. Phys. (2002).

[5] Zahn K. and Maret G., Phys. Rev. Lett., 82 (1999) 2721.

[6] Pesché R. and NÄGele G., Phys. Rev. E, 62 (2000) 5432.

[7] Nägele G., Phys. Rep., 272 (1996) 215.

[8] Härtl W., Wagner J., Beck Ch., Grierschner F. and Hempelmann R., J. Phys. Condens. Matter, 12 (2000) A287.

[9] NÄGele G. and Baur P., Europhys. Lett., 38 (1997) 557.

[10] Löwen H., Palberg T. and Simon R. G., Phys. Rev. Lett., 70 (1993) 1557.

[11] Löwen H., Phys. Rev. E, 53 (1996) R29.

[12] Ermak D. L. and McCammon J. A., J. Chem. Phys., 69 (1978) 1352.

[13] Dhont J. K. G., An Introduction to Dynamics of Colloids (Elsevier, Amsterdam) 1996.

[14] Beenakker C. W. J., J. Chem. Phys., 85 (1986) 1581. 